\title{
ACTITUDES, CONCEPCIONES Y PRÁCTICAS INCLUSIVAS DE DOCENTES Y EDUCADORES DIFERENCIALES DE ESCUELAS RURALES
}

Inclusive attitudes, conceptions and practices of teachers and differential educators of rural schools

\author{
ALEXIS ARAYA CORTÉS \\ Universidad Central de Chile \\ alexis.araya@ucentral.cl \\ YISELL ÁLVAREZ SANTANDER \\ Universidad Central de Chile \\ yisell.alvarez.s@gmail.com \\ LORETO GARÍN TIRADO \\ Universidad Central de Chile \\ loreto.garin465@gmail.com \\ FRANCISCA PALLERO BARAHONA \\ Universidad Central de Chile \\ franciscapallerob@gmail.com \\ NAYADETT PUCHI VALENZUELA \\ Universidad Central de Chile \\ nayadett.puchi@gmail.com \\ BÁRBARAVELIZ MALEBRÁN \\ Universidad Central de Chile \\ barbara.velizm@gmail.com
}

RESUMEN

El objetivo de la investigación fue describir las actitudes, concepciones y prácticas pedagógicas inclusivas de docentes y educadores diferenciales de establecimientos rurales de La Serena, Chile. Se utilizó un enfoque mixto de investigación con un diseño de carácter descriptivo. Se aplicó la Guía de Evaluación de Prácticas Inclusivas en el Aula (GEPIA) a un 
total de 49 profesores y para complementar la información se aplicó una entrevista semiestructurada a cinco docentes de aula y cinco educadores diferenciales. Los resultados cuantitativos evidenciaron que los docentes, se encuentran en una etapa intermedia de implementación de prácticas inclusivas. Los resultados cualitativos mostraron que ambos grupos, presentan una actitud positiva hacia el perfeccionamiento, planificación e implementación de prácticas inclusivas. Con relación a las concepciones, existen diferencias en el nivel de conocimiento sobre las prácticas inclusivas entre ambos grupos. Los docentes en general declaran una implementación de prácticas inclusivas, aunque reconocen una escasa formación con respecto a temas de inclusión y prácticas inclusivas específicas.

Palabras clave: Actitudes docentes; concepciones docentes; educación inclusiva; prácticas inclusivas.

\section{ABSTRACT}

The objective of the research was to describe inclusive attitudes, conceptions and practices in education, both in teachers and special educators, in rural settings in La Serena, Chile, by using a mixed research approach with a and a descriptive design. The Assessment Guide for Inclusive Practices in the Classroom (GEPIA for its acronym in Spanish) was applied to a total of 49 teachers, plus a semi-structured interview which was also applied to five Classroom Teachers and five Special Educators to collect the data needed for this research. Quantitative results revealed that teachers are at an intermediate level at the implementation of inclusive practices, while qualitative results showed that both groups present a positive attitude and good will towards self improvement, planning in the frame of inclusion, and the implementation of inclusive practices. With regard to conceptions, differences in the level of knowledge on inclusive practices between the two groups were evident. Though most teachers declared to apply inclusive practices in the classroom, they also accepted the fact that they had little training on topics related to inclusion and inclusive practices in the classroom.

Key words: Teaching attitudes; teaching conceptions; inclusive education; inclusive practices.

\section{PlanteAmieNTO DEL PROBLEMA}

Actualmente, la inclusión ha estado sujeta a grandes cambios en el ámbito educativo. En este sentido, Booth y Ainscow (2015) la consideran, en el ámbito educativo, como la participación de todos los estudiantes, señalando como uno de sus fines principales apoyar a los centros escolares para que sean más responsables ante la diversidad de su alumnado, 62 | INTEREDU № 3 VOL. II (DiciemBRE 2020) PÁGs. 61-81. ISSN: 2735-6523 
sea en razón a sus orígenes, intereses, experiencias, conocimientos y/o capacidades. Es por ello, que se considera relevante la elaboración de políticas que orienten a las instituciones educativas en la aplicación de acciones inclusivas.

Por consiguiente, el Ministerio de Educación (MINEDUC, 2009) bajo las diversas perspectivas de inclusión, en el año 2009 promulgó el Decreto Supremo N¹70, el cual en su artículo №86 establece como requisito la contratación de recursos humanos especializados, el trabajo colaborativo, la capacitación y la adquisición de recursos didácticos como apoyos fundamentales para el adecuado funcionamiento de los Programas de Integración Escolar (PIE). En la misma línea, en el año 2015 se comienza a implementar el Decreto $N^{\circ} 83$, el cual tiene como propósito definir criterios y orientaciones de adecuación curricular, que permitan planificar propuestas educativas de calidad para los estudiantes con necesidades educativas especiales de educación parvularia y educación básica que así lo requieran. A partir de estos criterios y orientaciones se garantiza la flexibilidad de las medidas curriculares para estos estudiantes, con el propósito de asegurar sus aprendizajes y desarrollo a través de la participación en propuestas educativas pertinentes y de calidad (MINEDUC, 2015).

En concordancia con las políticas anteriores, durante el año 2018 se promulgó el decreto №67 que aprueba normas mínimas nacionales sobre evaluación, calificación y promoción, derogando los decretos exentos N 511 de 1997, Nº 112 de 1999 y № 83 de 2001, todos del MINEDUC. Este decreto señala que los establecimientos educacionales deberán implementar las diversificaciones pertinentes para las actividades de aprendizaje y los procesos de evaluación de las asignaturas o módulos con los estudiantes que así lo requieran (MINEDUC, 2018).

Considerando la relevancia de la educación inclusiva, y especialmente de las prácticas pedagógicas inclusivas, la presente investigación es necesaria por las siguientes razones. En primer lugar, debido a que actualmente existen vacíos teóricos y de información sobre las actitudes y concepciones docentes, pues hay escasos estudios que permitan contextualizar respecto de la implementación de prácticas pedagógicas inclusivas en contextos rurales. En segundo lugar, se destaca la relevancia de las prácticas inclusivas en las escuelas, las que según Mateus et al. (2017), se consideran como una serie de atributos o características referidas a los conocimientos externos e internos de la comunidad educativa. Por lo tanto, estas características impulsan a alcanzar los objetivos curriculares propuestos y obtener resultados académicos esperados. Además, es relevante que en los establecimientos educacionales, el profesorado mantenga un pleno conocimiento sobre prácticas inclusivas y una adecuada actitud frente a su implementación, puesto que estas sirven para alcanzar una auténtica inclusión. 
En definitiva, se deben considerar las actitudes y concepciones que posean los docentes como un elemento fundamental para dar respuesta a la diversidad y por esto, es relevante conocer las actitudes, concepciones y prácticas inclusivas de docentes de aula regular y educadores diferenciales que implementan en establecimientos educativos rurales de la comuna de La Serena.

\section{ANTECEDENTES Y FUNDAMENTACIÓN TEÓRICA}

El desarrollo de la inclusión debe considerar una sociedad más equitativa, en la cual se respeten las diferencias, con la finalidad de beneficiar a todos y todas. Para Echeita (2017) la educación inclusiva tiene el propósito de cambiar los sistemas educativos, de modo que todos los estudiantes, sin excepción, puedan desarrollar plenamente su personalidad en el marco de un sistema educativo integral y común. En la misma línea, Booth y Ainscow (2015) señalan que dentro de una comunidad educativa deben existir tres dimensiones que se implementan e interactúan para llevar a cabo una educación inclusiva, considerando políticas, culturas y prácticas. Las políticas inclusivas se entienden como las normas establecidas las que se toman en cuenta para establecer según la Organización de las Naciones Unidas para la Educación (UNESCO, 2017) la igualdad en el disfrute del derecho a la educación de todas las personas. Las culturas inclusivas, según Mateus et al. (2017), están mediadas por el conjunto de creencias y valores con que sus miembros se identifican, los cuales, a su vez, orientan sus acciones. Finalmente, se consideran prácticas inclusivas a las diversas actividades y acciones, que movilizan recursos para promover la participación de todo el alumnado (Booth y Ainscow, 2015). Estás prácticas dependen del contexto en el que se desarrollan, teniendo en cuenta elementos como la heterogeneidad de los estudiantes, sus actitudes y las competencias del profesorado a la hora de innovar y de crear contextos de aprendizaje. En este sentido, las prácticas, reflejan las políticas y culturas inclusivas de las comunidades educativas (Mateus et al., 2017).

\subsection{APROXIMACIÓN A LAS PRÁCTICAS INCLUSIVAS.}

Los autores García, Romero y Escalante (2011) señalan que en el contexto de educación inclusiva el término prácticas inclusivas es usado para referirse a todo lo que hacen los centros educativos, considerando a las maestras y maestros, en su afán de brindar una atención educativa de calidad que beneficie a todos los estudiantes, sin exclusión. Entonces, una adecuada práctica inclusiva se debe entender como una actuación situada, que adquiere sentido y es viable a partir de una realidad concreta, de unos condicionantes estructurales que la hacen única e irrepetible (Marchesi, Durán, Giné y Hernández, 2009).

64 | INTEREDU № 3 VOL. II (DiciembRE 2020) PÁGS. 61-81. ISSN: 2735-6523 
Por otra parte, Flores, García y Romero (2017) afirman que las prácticas pedagógicas inclusivas se basan en la concepción de los derechos humanos, según la cual todos los ciudadanos tienen derecho a participar en todos los contextos y situaciones de la sociedad, logrando que la discriminación se reduzca o desaparezca. Con la intención de hacer efectivo este derecho sin discriminación y sobre la base de igualdad de oportunidades, es posible asegurar un sistema de educación inclusivo a todos los niveles, así como la enseñanza a lo largo de la vida.

Por último, las prácticas inclusivas educativas deben cumplir algunos requisitos, como: diseñar diversos materiales teniendo en cuenta las características del alumnado, usar diferentes estrategias de enseñanza-aprendizaje, flexibilidad curricular, libertad para que el alumnado decida qué y cuándo evidenciar su aprendizaje, ofrecer variedad de actividades que motiven al alumnado, con diferente grado de complejidad para que por sí mismas favorezcan la diversificación y organización óptima del tiempo (Rubio, 2015).

Con relación a estas prácticas inclusivas García et al. (2011) proponen considerar cinco subdimensiones: condiciones físicas del aula, planeación, metodología, uso del tiempo y evaluación.

En primera instancia, se considera como una de las prácticas inclusivas las condiciones físicas del aula, que de acuerdo con García et al. (2011) se refiere al uso y aprovechamiento de los recursos materiales en el aula, como material didáctico y de apoyo para brindar mejor atención a todos los alumnos y tener mayor diversidad de estrategias para la enseñanza, mobiliario y la ubicación favorable de este, de manera que todos tengan la misma accesibilidad. Por otra parte, García et al. (2011) definen el criterio de planeación como una práctica inclusiva, que se entiende como una organización previa por parte de los docentes respecto a las evaluaciones y las adecuaciones curriculares. De la misma forma, MINEDUC (2017) define el proceso de planificación o planeación, como un proceso sistémico y flexible en que se organizan y anticipan los procesos de enseñanza aprendizaje, con el propósito de orientar la práctica pedagógica en función de apoyar a los estudiantes. La tercera práctica inclusiva se relaciona con la metodología de enseñanza aprendizaje que, según García et al. (2011), es la forma en la que el docente presenta y organiza los contenidos. También se refiere a la manera en que el docente aborda los contenidos y busca desarrollar las competencias: si respeta la forma de aprender, si promueve el trabajo colaborativo, si realiza adecuaciones curriculares y si involucra al niño activamente en su aprendizaje. En lo que respecta al uso del tiempo, según García et al. (2011), se debe considerar la utilización y gestión del tiempo en relación con los ritmos de aprendizaje de cada estudiante. Asimismo, la Agencia de Calidad de la Educación (2018) determina que el ambiente de aula se caracteriza por un adecuado uso del tiempo, en el que 
se privilegian las horas lectivas por sobre otras actividades internas y externas a la clase. Finalmente, los autores refieren a la subdimensión de evaluación como un factor relevante para la implementación de prácticas inclusivas, en el cual García et al. (2011) lo definen como una forma y proceso de evaluar los aprendizajes respetando el ritmo de cada estudiante.

\subsection{ACTITUDES Y CONCEPCIONES EDUCATIVAS.}

Las actitudes y concepciones son dos conceptos relevantes asociados a las prácticas inclusivas que permiten una adecuada implementación en el aula. Por una parte, las actitudes son entendidas como "un conjunto de percepciones, creencias, sentimientos a favor o en contra y formas de reaccionar ante la postura educativa que centra su esfuerzo en el logro de los aprendizajes de todos los estudiantes" (Granada, Pómes, y Sanhueza, 2013, p. 53). Además, las autoras mencionan que la actitud del profesor es fundamental en el proceso de inclusión educativa, entendiéndose por actitud una posición u orientación del pensamiento, que se traduce en una forma determinada de pensar, actuar o reaccionar.

Por otra parte, los factores que influyen en una actitud positiva frente a la inclusión educativa según Chiner (2011) se centran en el contexto, es decir, integrar las variables ambientales que sean capaces de generar cambios en la percepción docente. Para ello, es necesario la reestructuración y el ordenamiento de todo el sistema. Cabe destacar, que la mala ejecución de las prácticas educativas se puede deber a un "temor a enfrentar nuevos desafíos y desconocimiento a las nuevas prácticas inclusivas" (Díaz y Franco, 2010, p. 26).

Las concepciones docentes en esta investigación serán entendidas como los saberes o conocimientos que posee el profesor. Como menciona Angulo (2012), cualquiera que sea la situación y el contexto, el docente en su quehacer se conocerá como experto, debido a que aplica el conocimiento teórico -generado por la investigación y perfeccionamientos- y siempre entendiendo que hay una diferencia entre lo que sabe el docente y lo que lleva a la práctica.

Asimismo, Espinosa y Valdebenito (2016) mencionan que una de las razones para indagar las concepciones de los docentes se debe a la influencia y relación que estas poseen en las prácticas pedagógicas. Sin embargo, las diferentes concepciones que presentan los docentes no están dadas por las características personales o individuales, sino por variables contextuales y de trayectoria profesional que tienen una relación directa con esos cambios que se están produciendo en el sistema educativo, existiendo una clara relación entre concepciones de la docencia y prácticas docentes. Sin embargo, esta relación está mediatizada por el contexto organizacional en el cual trabaja el profesional (Molpeceres, Chulvi y Bernad, 2004). 
Considerando los elementos teóricos abordados, el presente estudio propone dos objetivos. Como primer objetivo, se plantea describir las prácticas inclusivas pedagógicas reportadas por docentes de establecimientos rurales de la comuna de La Serena. El segundo objetivo busca describir las actitudes y concepciones de los docentes y educadores diferenciales sobre las prácticas inclusivas pedagógicas.

\section{Metodología}

\subsection{ENFOQUE Y DISEÑO DEL ESTUDIO.}

El estudio se realizó con un enfoque mixto, el cual considera un conjunto de procesos sistemáticos, empíricos y críticos de investigación e implican la recolección y el análisis de datos cuantitativos y cualitativos (Hernández, Fernández y Baptista, 2014; Ruiz, Borboa y Rodríguez 2013). En este sentido Creswell (2009) determina que los datos cualitativos y cuantitativos pueden ser fusionados en una larga base de datos o los resultados usados lado a lado para reforzar uno al otro. El diseño de investigación utilizado es descriptivo, Abreu (2012) menciona que la investigación descriptiva se ajusta al enfoque mixto.

\subsection{PARTICIPANTES.}

La muestra del estudio estuvo compuesta por 49 profesionales del área de la educación, específicamente, por profesores de aula regular y educadores diferenciales. La totalidad de los profesores se encontraba trabajando en educación preescolar y básica en establecimientos rurales con PIE de la comuna de La Serena. En la Tabla 1 se detallan las características de la muestra total y en la Tabla 2 los profesores que participaron en la entrevista. La selección de los participantes fue mediante un muestreo por conveniencia, debido a que se seleccionaron aquellos casos accesibles que acepten ser incluidos en el estudio (Otzen y Manterola, 2017).

Tabla 1. Antecedentes de los participantes totales del estudio.

\begin{tabular}{lcccc}
\hline \multirow{2}{*}{$\begin{array}{c}\text { Establecimientos } \\
\text { educacionales }\end{array}$} & \multicolumn{2}{c}{ Formación de base } & Cantidad de & Porcentaje \\
\cline { 2 - 3 } & $\begin{array}{c}\text { Profesor(a) de } \\
\text { Ed. Diferencial }\end{array}$ & $\begin{array}{c}\text { Profesor(a) de } \\
\text { Educación } \\
\text { Regular }\end{array}$ & participantes & \\
\hline Colegio Lambert & 1 & 6 & 7 & $14,3 \%$ \\
Colegio Caleta San Pedro & 3 & 9 & 12 & $24,5 \%$ \\
Colegio Intercultural & 2 & 7 & 9 & $18,4 \%$ \\
Colegio Alfalfares & 3 & 7 & 10 & $20,4 \%$ \\
Colegio Saturno & 3 & 8 & 11 & $22,4 \%$ \\
\hline Totales & 12 & 37 & 49 & $100 \%$
\end{tabular}

Fuente: Elaboración propia. 
Tabla 2. Antecedentes de los participantes entrevistados.

\begin{tabular}{lcccc}
\hline \multicolumn{1}{c}{$\begin{array}{c}\text { Establecimientos } \\
\text { educacionales }\end{array}$} & \multicolumn{2}{c}{ Formación de base } & $\begin{array}{c}\text { Cantidad de } \\
\text { participantes }\end{array}$ & Porcentaje \\
\cline { 2 - 3 } & $\begin{array}{c}\text { Profesor(a) de } \\
\text { Ed. Diferencial }\end{array}$ & $\begin{array}{c}\text { Profesor(a) de } \\
\text { Educación Regular }\end{array}$ & & \\
\hline Colegio Lambert & 1 & 1 & 2 & $20 \%$ \\
$\begin{array}{l}\text { Colegio Caleta San } \\
\text { Pedro }\end{array}$ & 1 & 1 & 2 & $20 \%$ \\
Colegio Intercultural & 1 & 1 & 2 & $20 \%$ \\
Colegio Alfalfares & 1 & 1 & 2 & $20 \%$ \\
Colegio Saturno & 1 & 1 & 2 & $20 \%$ \\
\hline Totales & 5 & 5 & 10 & $100 \%$ \\
\hline
\end{tabular}

Fuente: Elaboración propia.

\subsection{INSTRUMENTOS.}

Para la recogida de la información se seleccionaron y aplicaron dos instrumentos. En la etapa cuantitativa se utilizó la Guía de Evaluación de Prácticas Inclusivas en el Aula (GEPIA) elaborada por García et al. (2011). Para efectos de esta investigación se aplicó la modalidad de auto-reporte de tipo escala de Likert, considerando solo la dimensión de prácticas pedagógicas inclusivas. La dimensión está compuesta por 27 reactivos y cinco subdimensiones: condiciones físicas del aula, planeación, uso del tiempo, metodología y evaluación. La consistencia interna del instrumento es de .92, lo que indica una alta confiabilidad.

En la etapa cualitativa, para recoger información sobre las actitudes y concepciones de los docentes sobre las prácticas inclusivas se utilizó la entrevista semiestructurada. Esta técnica es considerada por Canales (2006) como la comunicación interpersonal establecida entre el investigador y el sujeto de estudio, a fin de obtener respuestas verbales a las interrogantes planteadas sobre el problema propuesto. Asimismo, este tipo de entrevista se asocia con la expectativa de que es más probable que los sujetos entrevistados expresen sus puntos de vista de manera relativamente abierta, que en una entrevista estandarizada o un cuestionario (Flick, 2007).

El guion de la entrevista fue validado a través del juicio de expertos de tres académicos con grado de magíster y con una experiencia de al menos cinco años en docencia universitaria, quienes revisaron y aprobaron dicho instrumento. El guión estaba compuesto 
por un conjunto de categorías y subcategorías generadas a priori desde la teoría, que se presentan en la Tabla 3.

Tabla 3. Categorías y subcategorías de la investigación.

\begin{tabular}{ll}
\hline \multicolumn{1}{c}{ Categorías } & \multicolumn{1}{c}{ Subcategorías } \\
\hline Actitudes sobre las prácticas inclusivas & $\begin{array}{l}\text { Perfeccionamiento docente } \\
\text { Planificaciones } \\
\text { Implementación de prácticas inclusivas }\end{array}$ \\
Concepciones sobre las prácticas inclusivas & Conceptualización \\
\hline
\end{tabular}

Fuente: Elaboración propia.

\subsection{PROCEDIMIENTO.}

En primer lugar, se solicitó la autorización a los/los directores/as de cada establecimiento educacional para realizar el estudio. Posteriormente, se presentaron los objetivos y características de la investigación a los docentes quienes participaron de forma voluntaria y firmaron un documento de consentimiento informado, en el cual se explicitó el carácter confidencial de la información y la ausencia de riesgos para los encuestados y entrevistados.

De acuerdo con una programación con las cinco escuelas rurales, las investigadoras aplicaron ambos instrumentos de recogida de información en un mismo momento. Primero se aplicó la Guía de Evaluación de Prácticas Inclusivas en el Aula (GEPIA) a todos los docentes del establecimiento. Luego, se realizaron de forma individual las entrevistas a docentes y educadores/as diferenciales.

\subsection{ANÁLISIS DE DATOS.}

Para los análisis de la etapa cuantitativa, se empleó el programa estadístico SPSS, versión 20.0. En primer lugar, se realizó un análisis descriptivo de tendencia central y de variabilidad para cada una de las variables. Posteriormente, se realizó un análisis de tablas de contingencia para resumir la información y establecer la asociación entre las variables. Con relación a la etapa cualitativa, se realizó un análisis de las narrativas de cada miembro de la comunidad educativa usando el método de análisis de contenido. Se llevó cabo el análisis que propone Rodríguez, Gil y García (1999), efectuando tres acciones 
fundamentales: reducción de datos; disposición y transformación de datos, y obtención y verificación de conclusiones. Además, se utilizó una matriz de análisis para apoyar el proceso, debido a la posibilidad de identificar las codificaciones de cada investigador y de obtener una visualización de las categorías y subcategorías.

\section{RESUlTADOS}

\subsection{PRÁCTICAS PEDAGÓGICAS INCLUSIVAS.}

Respecto a la implementación de prácticas pedagógicas inclusivas reportadas por los participantes, se observa en la Tabla 4, que todas las subdimensiones tuvieron un promedio superior a la media teórica esperada $(\mathrm{M}$ [teórico] $=1.5)$ en una escala de 0 a 3 puntos. La puntuación más alta fue para las subdimensiones de Condiciones físicas del aula $(\mathrm{M}=2.6$; $\mathrm{DS}=$ $0.4)$, Uso del tiempo $(\mathrm{M}=2.6$; $\mathrm{DS}=0.4)$, Metodología $(\mathrm{M}=2.6$; $\mathrm{DS}=0.4)$ y Evaluación $(\mathrm{M}=2.6$; $\mathrm{DS}=0.5)$. Dentro de las puntuaciones bajas se encuentra la subdimensión de Planeación $(\mathrm{M}=$ 2.5; DS = 0.4). Los puntajes promedios indican que los profesores y educadores diferenciales declararon un alto nivel de utilización de estas acciones en el aula ( $\mathrm{M}=2.6$; $\mathrm{DS}=0.4)$.

Tabla 4. Promedio de las prácticas pedagógicas inclusivas implementadas en el aula.

\begin{tabular}{lcccc}
\hline \multicolumn{1}{c}{ Subdimensiones } & Mínimo & Máximo & Media & Desv. típ. \\
\hline Condiciones físicas del aula & 1 & 3 & 2.6 & 0.4 \\
Planeación & 2 & 3 & 2.5 & 0.4 \\
Uso del tiempo & 2 & 3 & 2.6 & 0.4 \\
Metodología & 2 & 3 & 2.6 & 0.4 \\
Evaluación & 1 & 3 & 2.6 & 0.5 \\
\hline Total & 1 & 3 & 2.6 & 0.4 \\
\end{tabular}

Fuente: Elaboración propia.

Después de realizar el análisis descriptivo de las diferentes subdimensiones que componen la dimensión de prácticas pedagógicas inclusivas del cuestionario GEPIA, se realizó un análisis de tablas de contingencia. Según lo reportado por los participantes, en la Tabla 5 se observa que todas las subdimensiones obtienen un alto porcentaje en el uso de prácticas inclusivas, encontrándose en la valoración siempre el porcentaje más alto para Condiciones físicas del aula (71.4\%) y el más bajo para Uso del tiempo (61.2\%). Al analizar los porcentajes globales de las prácticas pedagógicas inclusivas, un $66.5 \%$ de los profesores 70 | INTEREDU № 3 VOL. II (DiCIEMBRE 2020) PÁGS. 61-81. ISSN: 2735-6523 
declara utilizarlas siempre, mientras que un 32.5\%, señala utilizarlas casi siempre y con un porcentaje menor de $1 \%$ algunas veces.

Tabla 5. Distribución de las prácticas pedagógicas inclusivas según las valoraciones de los participantes.

\begin{tabular}{|c|c|c|c|c|}
\hline Subdimensiones & Algunas veces & Casi siempre & Siempre & Total \\
\hline \multirow{2}{*}{$\begin{array}{l}\text { Condiciones físicas } \\
\text { del aula }\end{array}$} & 1 & 13 & 35 & 49 \\
\hline & $2 \%$ & $26.5 \%$ & $71.4 \%$ & $100 \%$ \\
\hline \multirow[t]{2}{*}{ Planeación } & 0 & 18 & 31 & 49 \\
\hline & $0 \%$ & $36.7 \%$ & $63.3 \%$ & $100 \%$ \\
\hline \multirow[t]{2}{*}{ Uso del tiempo } & 0 & 19 & 30 & 49 \\
\hline & $0 \%$ & $38.8 \%$ & $61.2 \%$ & $100 \%$ \\
\hline \multirow[t]{2}{*}{ Metodología } & 0 & 15 & 34 & 49 \\
\hline & $0 \%$ & $30.6 \%$ & $69.4 \%$ & $100 \%$ \\
\hline \multirow[t]{2}{*}{ Evaluación } & 1 & 15 & 33 & 49 \\
\hline & $2 \%$ & $30.6 \%$ & $67.3 \%$ & $100 \%$ \\
\hline \multirow[t]{2}{*}{ Total } & 2 & 80 & 163 & 245 \\
\hline & $1 \%$ & $32.5 \%$ & $66.5 \%$ & $100 \%$ \\
\hline
\end{tabular}

Fuente: Elaboración propia.

Como se observa en la Tabla 6, la totalidad del profesorado de escuelas rurales evidencia porcentajes sobre el $70 \%$ en el grado de acuerdo en las cinco subdimensiones asociadas a la variable prácticas pedagógicas inclusivas. En cuanto a lo declarado por los participantes, se puede observar que en todas las subdimensiones, el Colegio Alfalfares supera el grado de acuerdo al compararlo con el resto de las escuelas. En la subdimensión de Condiciones físicas del aula, el Colegio Caleta San Pedro es el que evidenció un menor grado de acuerdo (79\%), mientras que en la subdimensión de Planeación (73\%), Uso del tiempo (78\%) y Evaluación (71\%), el Colegio Lambert evidenció un menor porcentaje en el grado de acuerdo. Por último, en la subdimensión Metodología, los Colegios Caleta San Pedro y Lambert evidenciaron un menor grado de acuerdo (80\%) que el resto de las escuelas rurales.

Tabla 6. Grado de acuerdo en porcentaje según agrupación por colegio por cada una de las dimensiones de la variable prácticas pedagógicas inclusivas.

\begin{tabular}{|c|c|c|c|c|c|c|}
\hline Subdimensiones & & & $\begin{array}{l}\text { Colegio Caleta } \\
\text { San Pedro }\end{array}$ & $\begin{array}{c}\text { Colegio } \\
\text { Intercultural }\end{array}$ & $\begin{array}{c}\text { Colegio } \\
\text { Alfalfares }\end{array}$ & $\begin{array}{l}\text { Colegio } \\
\text { Saturno }\end{array}$ \\
\hline $\begin{array}{l}\text { Condiciones físicas } \\
\text { aula }\end{array}$ & del & $80 \%$ & $79 \%$ & $90 \%$ & $94 \%$ & $85 \%$ \\
\hline Planeación & & $73 \%$ & $84 \%$ & $90 \%$ & $95 \%$ & $74 \%$ \\
\hline Uso del tiempo & & $78 \%$ & $81 \%$ & $86 \%$ & $99 \%$ & $85 \%$ \\
\hline
\end{tabular}




\begin{tabular}{lccccc}
\hline Metodología & $80 \%$ & $80 \%$ & $93 \%$ & $96 \%$ & $87 \%$ \\
\hline Evaluación & $71 \%$ & $83 \%$ & $89 \%$ & $98 \%$ & $83 \%$ \\
\hline Promedio total & $76 \%$ & $81 \%$ & $90 \%$ & $96 \%$ & $83 \%$ \\
\hline
\end{tabular}

Fuente: Elaboración propia.

\subsection{ACTITUDES SOBRE LAS PRÁCTICAS INCLUSIVAS.}

En este apartado se desprenden tres subcategorías de análisis: Perfeccionamiento docente, planificaciones e implementación de Prácticas Pedagógicas Inclusivas.

\subsubsection{PERFECCIONAMIENTO DOCENTE}

Según los/as educadores diferenciales entrevistados, el sistema educativo aún no está totalmente preparado para enfrentar la inclusión. Sin embargo, denotan un avance con el transcurso del tiempo, el cual está en vías de mejoramiento, puesto que antiguamente la inclusión no era un tema recurrente. Asimismo, señalan que el sistema educativo ha dado mayor énfasis a lo administrativo, pero no se enfocan en la preparación de los docentes para enfrentar la inclusión o en aspectos que afectan directamente el proceso de enseñanza aprendizaje.

La totalidad de los entrevistados presentan una disposición positiva frente a las nuevas prácticas inclusivas. En primera instancia, los/as educadores diferenciales señalaron que su foco es privilegiar las prácticas inclusivas, implementando estrategias como el Diseño Universal para el Aprendizaje (DUA), trabajar con material concreto y trabajar en codocencia. De igual manera, los docentes de aula regular señalaron tener una buena disposición, puesto que el sistema educativo está sometido a cambios y ellos deben tener una mirada más amplia de cómo abordar la inclusión educativa. Ambos grupos consideraron necesario perfeccionarse en nuevas prácticas inclusivas, ya que siempre están surgiendo nuevas metodologías y estrategias. Además, declararon no sentirse totalmente preparados y actualizados en temas específicos de atención a la diversidad.

Educador/a diferencial 4: "El sistema educativo chileno se enfoca mucho en el tema de administración, uno que acá tiene programa de integración es mucho tema de capacitación hacia la administración, pero muy poco al quehacer educativo... de cómo trabajar, de cómo resolver conflictos, de cómo enfrentarnos a los distintos ritmos y estilos de aprendizaje de los niños... Eh, no está, o sea, de un cien por ciento no está, yo creo que faltan años todavía, pero ya estamos en un 60 yo creo, ya hemos avanzado".

Docente de aula regular 1: “Mira, yo creo que el sistema no está preparado para enfrentar la inclusión desde la formación de pregrado. Te puedo hablar desde mi 72 | INTEREDU № 3 VOL. II (DiCIEMBRE 2020) PÁGS. 61-81. ISSN: 2735-6523 
concepción, yo salí de la universidad hace seis años y atender a la diversidad no era un tema recurrente en la universidad...".

\subsubsection{PLANIFICACIONES}

De acuerdo a lo mencionado por los/as educadoras diferenciales, las planificaciones deberían incorporar prácticas inclusivas, puesto que permiten la diversificación en el aula. Además, hicieron referencia principalmente al DUA y la codocencia como estrategias que favorecen la implementación de prácticas inclusivas en el aula. Los docentes de aula concordaron con lo señalado anteriormente. No obstante, señalaron que trabajan con cursos numerosos y disponen de poco tiempo. Por lo tanto, es complejo considerar estas prácticas inclusivas en las planificaciones, aunque que finalmente las implementan con el apoyo de los/as educadoras diferenciales.

Con relación a las respuestas educativas que entregan a los estudiantes, ambos grupos de profesores señalaron que, según sus experiencias personales, trabajan en el establecimiento desde un enfoque inclusivo. Por lo tanto, coincidieron en que existe una coordinación entre los actores de la comunidad educativa, en donde acuerdan horarios de trabajo colaborativo. Cabe mencionar, que los docentes de aula consideran al educador/a diferencial como un actor importante e influyente para desarrollar planificaciones desde una mirada inclusiva.

Educador/a diferencial 1: “...Que se estén haciendo estas prácticas inclusivas y porque es importante, porque es un trabajo que se realiza colaborativo con el profesor, nosotros hacemos codocencia, somos uno, estamos los dos al mismo nivel, estamos los dos en un sentido de paridad. Entonces es importante también, porque todo eso, los conocimientos del profesor y los conocimientos de los educadores diferenciales se hace una riqueza de aprendizaje muy muy linda para los niños".

Docente de aula regular 2: "Nosotros trabajamos en equipo, pero trabajamos conversando por, o sea, de cada niño, y vemos de qué manera abordar ese caso, por ejemplo, así de esa forma nosotros estamos trabajando. Que es el trabajo colaborativo que yo te digo del programa de integración que tenemos con las niñas".

\subsubsection{IMPLEMENTACIÓN DE PRÁCTICAS INCLUSIVAS}

Según las docentes entrevistadas se deberían implementar prácticas inclusivas en el aula, ya que todos los estudiantes presentan alguna necesidad educativa. Las acciones se deben implementar a través de actividades lúdicas, grupos interactivos, material concreto y considerando el DUA. Se logra evidenciar una segunda mirada por parte de los docentes de aula, debido a que mencionaron que las prácticas inclusivas no son implementadas por desconocimiento o pereza, sino que requieren de tiempo y trabajo adicional.

INTEREDU № 3 VoL. II (DICIEMBRE 2020) PÁGS. 61-81. ISSN: 2735-6523| 73 
Asimismo, los/as educadores/as diferenciales señalaron que para enfrentar la atención a la diversidad en el aula es necesario utilizar estrategias diversificadas, tales como: grupos interactivos, juegos, material concreto, trabajo colaborativo y codocencia, focalizándose en los estudiantes con necesidades educativas especiales.

Docente de aula regular 2: "Trabajando en forma personalizada, con cada niño, y son todos distintos. Eh, entonces es agotador, cansa, porque hay que trabajar con ellos, con cada uno".

Educador/a diferencial 1: "Siento que es importante que nosotros tengamos prácticas inclusivas al momento de adecuar una actividad, al momento de diversificar una evaluación, al momento de enseñar una forma mucho más lúdica que abarque a todos los niños".

\subsection{CONCEPCIONES SOBRE LAS PRÁCTICAS INCLUSIVAS.}

En esta categoría se desprenden dos subcategorías: conceptualización y prácticas pedagógicas inclusivas.

\subsubsection{CONCEPTUALIZACIÓN}

Para los educadores diferenciales la inclusión significa brindar las mismas oportunidades a todos/as los estudiantes, para así entregar un aprendizaje de calidad y de igual manera a cada estudiante. Teniendo en consideración que todos los estudiantes son diferentes, presentan diversas necesidades y aprenden de diversas formas. Para los docentes de aula regular, la inclusión de igual manera es brindarles las mismas oportunidades a todos los estudiantes, desde la mirada de la integración escolar. En donde ellos como docentes de aula regular deben tener las herramientas y crear un ambiente adecuado para un óptimo aprendizaje.

En cuanto a los conocimientos de educadores diferenciales, las prácticas inclusivas se relacionan con las estrategias tales como trabajo colaborativo, estrategias diversificadas, coenseñanza, plan de apoyo individual (PAI) y plan de adecuación curricular individual (PACI). Los docentes de aula regular presentan respuestas bastantes similares, basándose en la utilización del DUA y el apoyo del PIE.

Educador/a diferencial 2: “...Lo mismo que le nombraba, el trabajo colaborativo, las estrategias diversificadas, co-enseñanza, estrategias efectivas, PAI - PACI, eso, estrategias más que nada inclusivas".

74 | INTEREDU № 3 VOL. II (DiCIEMBRE 2020) PÁGS. 61-81. ISSN: 2735-6523 
Docente de aula regular 3: “...Bueno, las prácticas como prácticas son estrategias que utilizamos los profesores para poder llevar a cabo un trabajo más dirigido con los estudiantes y eso está apoyado con la educadora PIE, ya que son las especialistas, digamos, en esta área".

\subsubsection{PRÁCTICAS INCLUSIVAS}

Según las educadoras diferenciales, las prácticas inclusivas que implementan dentro del aula son estrategias diversificadas, además de realizar adecuaciones curriculares y trabajar en conjunto con las docentes de aula regular en co-enseñanza. Sin embargo, los docentes de aula regular evidenciaron que las prácticas inclusivas se implementan de maneras distintas, desde el proyecto educativo hasta realizar modelamientos, diversos trabajos y estrategias con los estudiantes que presenten necesidades educativas especiales.

Según las entrevistadas, las prácticas inclusivas más efectivas son el uso del material concreto, ya que de esta manera se facilita el aprendizaje y el trabajo colaborativo, específicamente la co-enseñanza que se realiza dentro de aula.

Educador/a diferencial 1: “Las prácticas inclusivas generalmente son adecuaciones curriculares, son diversificación curricular, también son estrategias como las preguntas de Raphael".

Docente de aula regular 1: “Que yo siento que es más efectiva es el proyecto Roma, ya que está centrado en el pensamiento y reflexión del estudiante en un modelo de aprendizaje por descubrimientos, lo que nos permite que el niño pueda él mismo construir su aprendizaje".

\section{DISCUSIÓN DE RESULTADOS}

El presente estudio buscó describir las actitudes, concepciones y prácticas pedagógicas inclusivas que declaran los docentes y educadores diferenciales de establecimientos rurales de la comuna de La Serena.

De acuerdo con los resultados cuantitativos, se logra evidenciar que la mayoría de los docentes reportaron utilizar prácticas pedagógicas inclusivas en el aula. Estos hallazgos son similares a los encontrados por Hernández y García (2017), en su estudio los docentes reportan altas prácticas inclusivas. Sin embargo, hay temores y preocupaciones para llevar a cabo estas prácticas en el aula. Asimismo, el estudio realizado por García, Romero, Rubio, Jocabed y Martínez (2015), afirma que los profesores tienen prácticas altamente favorecedoras a la inclusión, ya que las puntuaciones en la GEPIA de auto-reporte, son altas. Los resultados ponen de manifiesto que el nivel de prácticas inclusivas que usan 
habitualmente en las aulas los profesores es tendente a la inclusión (Gómez Castillo, Camacho, Sánchez y De La Peña, 2017).

Sin embargo, los establecimientos precisan de mayores apoyos en relación con las planificaciones, lo cual refleja la necesidad de flexibilizar el currículum y prestar atención al desarrollo organizativo como una estrategia de cambio que surge desde el interior de la escuela y se ajusta a las necesidades de esta, donde el profesorado tiene el compromiso y derecho de proponer y hacer los cambios pertinentes y beneficiar al alumnado (Sales et al., 2010).

Respecto de los resultados cualitativos, se evidenció que la totalidad de docentes entrevistados presentan una actitud positiva hacia el perfeccionamiento docente, planificación e implementación de prácticas pedagógicas inclusivas. Estos hallazgos son similares a los encontrados en el estudio realizado por Rayo y Pereyra (2015), quienes señalan que las actitudes ante prácticas inclusivas de los docentes son inclusivas y adecuadas.

Respecto a las concepciones, se observa una incongruencia respecto al conocimiento que poseen docentes y educadores diferenciales sobre las prácticas inclusivas. Además, se observa poca claridad por parte de la mayoría de los entrevistados, específicamente sobre conceptos asociados a inclusión y prácticas pedagógicas inclusivas, siendo una barrera que dificulta la articulación entre gestión, trabajo y educación (Castillo, 2010). Estos resultados se pueden contrastar con los encontrados por Hernández y García (2017), quienes señalan que los profesores denotan preocupación de no tener los conocimientos, ni las habilidades requeridas para enseñar a los estudiantes con discapacidad en escuelas regulares. Asimismo, Flores et al. (2017), identificaron la necesidad de un plan de actualización docente que enriquezca conceptualmente al profesorado y propicie la implementación de la educación inclusiva dentro de las escuelas regulares.

\section{CONCLUSIONES}

A partir de los resultados respecto de las actitudes, concepciones y prácticas pedagógicas inclusivas reportadas por el profesorado, se pueden extraer varias conclusiones. En primer lugar, en relación a la implementación de prácticas inclusivas, se concluye que las educadoras diferenciales y docentes de aula regular declaran que dichas acciones se implementan en el aula. Lo anterior, debido a que las subdimensiones de condiciones físicas del aula, planeación, uso del tiempo, metodología y evaluación se encuentran sobre la media teórica esperada. Sin embargo, la subdimensión de planeación es la que resulta con una media más baja que el resto, lo que podría indicar que los docentes implementan prácticas inclusivas, pero no las incorporan detalladamente en sus 76 | INTEREDU № 3 VOL. II (DiCIEMBRE 2020) PÁGS. 61-81. ISSN: 2735-6523 
planificaciones, resolviéndose de manera informal en el aula. Con este estudio se comprueba nuevamente, que los docentes declaran una alta utilización de prácticas inclusivas en sus aulas, pero a la vez, señalan una escasa formación con respecto a temas de inclusión y prácticas pedagógicas inclusivas específicas para responder a la diversidad y necesidades educativas especiales de los estudiantes.

En segundo lugar, se concluye que tanto los profesores de aula como los educadores diferenciales presentan una actitud positiva hacia las prácticas pedagógicas inclusivas, en relación con el perfeccionamiento docente, planificación e implementación en el aula. No obstante, a pesar de mantener una disposición positiva, se denota una desmotivación asociada al tiempo, la demanda de trabajo extraordinario y los asuntos administrativos, que conlleva a que dichas prácticas en algunas ocasiones no sean ejecutadas, lo que constituye una barrera importante para la implementación de prácticas inclusivas. Asimismo, las educadoras diferenciales están dispuestas e interesadas en innovar implementando nuevas prácticas que abarquen las necesidades de cada estudiante, considerando y reconociendo el DUA como principal estrategia para responder a la diversidad de estudiantes y a las necesidades educativas especiales. Este grupo de profesoras destaca que el perfeccionamiento docente es crucial para llevar a cabo una plena inclusión.

Por último, las concepciones que poseen los docentes de aula regular y educadores diferenciales sobre las prácticas inclusivas no concuerdan completamente, puesto que los docentes de aula regular no son conceptualmente precisos con los términos asociados a las prácticas inclusivas. Por el contrario, los educadores diferenciales poseen un conocimiento más amplio sobre la temática. Es importante, destacar que las concepciones son entendidas, según Angulo (2012), como cualquier situación o contexto vivenciado en que el docente en su quehacer se conoce como experto técnico, puesto que aplica su conocimiento teórico.

De este modo, los docentes entrevistados señalaron que las prácticas inclusivas se relacionan con las estrategias implementadas en el aula y el trabajo que se lleva a cabo para dar respuesta a las necesidades educativas de todo el estudiantado. Por lo que se concluye que los profesores consideran como prácticas pedagógicas inclusivas fundamentales el trabajo colaborativo, la codocencia y el DUA.

Cabe mencionar, que una de las categorías de estudio tuvo relación con el concepto de inclusión educativa. En este sentido, los docentes evidenciaron una clara definición que consistía en el trabajo con todos y todas las estudiantes, sin hacer distinciones, considerando las necesidades y oportunidades aprendizaje de todo el alumnado. Sin embargo, algunos docentes de aula regular comprenden la inclusión como una ley que el sistema educativo ha ido modificando y mejorando en torno a la integración escolar. Asimismo, señalan que para lograr una inclusión educativa los docentes deben tener noción acerca de las cualidades 
del grupo curso, considerando el ritmo, estilo y canales de aprendizaje de todos los estudiantes.

Por último, los hallazgos expuestos ponen de manifiesto la necesidad de abordar en las escuelas rurales temáticas asociadas a las prácticas pedagógicas inclusivas como elemento clave y crucial de respuesta a la diversidad de estudiantes. A pesar de que las escuelas reportan un alto nivel de implementación de estas acciones, es necesario profundizar en la conceptualización, diversificación y sistematización de prácticas inclusivas.

\section{LIMITACIONES DEL ESTUDIO Y PROSPECTIVA}

En la actualidad, la inclusión y las prácticas pedagógicas inclusivas en aula se consideran una línea de investigación indiscutiblemente relevante. En este sentido, es preciso señalar ciertas limitaciones del presente estudio. En primer lugar, los resultados refieren a establecimientos rurales y a un número reducido de profesores, por lo cual no pueden ser generalizados a contextos urbanos $u$ otros grupos de profesores. En segundo lugar y como consecuencia del instrumento de auto-reporte utilizado, solo se obtuvo información proporcionada por los mismos participantes y generalmente este tipo de instrumentos muestran altas valoraciones. Por tanto, es necesario que en futuros estudios se incremente la muestra de profesores y asistentes de la educación que apoyan el proceso de inclusión en las escuelas, incorporar colegios urbanos, comparar las prácticas pedagógicas inclusivas con otras escuelas y aplicar el instrumento GEPIA en modalidad de observación en aula para contrastar la auto-percepción del profesorado.

\section{REFERENCIAS BIBLIOGRÁFICAS}

Abreu, J. (2012). Hipótesis, método y diseño de investigación. Daena: Revista Internacional de Buena Conciencia. http://www.spentamexico.org/v7-n2/7(2)187-197.pdf

Agencia de Calidad de la Educación (2018). Estrategias pedagógicas y rutinas para el desarrollo de aprendizajes significativos. https://www.agenciaeducacion.cl/wpcontent/uploads/2018/02/Reporte_RBD_4456.pdf

Angulo, G. (2012). Las concepciones sobre el docente o diferentes maneras de concebir el ejercicio de la docencia. Revista de Investigación, 36(75),11-31. https://www.redalyc.org/articulo.oa?id=3761/376140390001

Booth, T., y Ainscow, M. (2015). Guía para la evaluación inclusiva, desarrollando el aprendizaje y la participación en los centros escolares. (Adaptación de la 3a edición revisada del Index for Inclusion). Traducción y adaptación por Echeita, G., Muñoz, Y., Simón, C., y

78 | INTEREDU № 3 VOL. II (DiCIEMBRE 2020) PÁGS. 61-81. ISSN: 2735-6523 
Sandoval, M. Madrid, España: FUHEM, OEI. https://www.oei.es/historico/IndexLibroAgosto.pdf

Canales, M. (2006) Metodologías de la investigación social. Santiago: LOM Ediciones.

Castillo, M. (2010). La profesión docente. Revista médica de Chile, 138(7), 902-907. https://dx.doi.org/10.4067/S0034-98872010000700017

Chiner, E. (2011). Las percepciones y actitudes del profesorado hacia la inclusión del alumnado con necesidades educativas especiales como indicadores del uso de prácticas educativas inclusivas en el aula (Tesis doctoral). Universidad de Alicante, Alicante. http://hdl.handle.net/10045/19467

Creswell, J. (2009). El diseño y la realización de la investigación de métodos mixtos. Los Ángeles: SAGE.

Díaz, O., y Franco, F. (2010). Percepción y actitudes hacia la inclusión educativa de los docentes de Soledad, Atlántico (Colombia), 2008. Zona Próxima, (12) https://search.proquest.com/docview/1435672229?accountid=39719

Echeita, G. (2017). Educación inclusiva: Sonrisas y lágrimas. Aula Abierta, 46 (2), 17-24. https://doi.org/10.17811/rifie.46.2017.17-24

Espinosa, J., y Valdebenito, V. (2016). Explorar las concepciones de los docentes respecto al proceso de educación inclusiva para la mejora institucional. Revista latinoamericana de educación inclusiva, 10(1), 195-213. https://dx.doi.org/10.4067/S071873782016000100010

Flick, U. (2007). Introducción a la investigación cualitativa. Madrid: Ediciones Morata.

Flores, V., García, I., y Romero, S. (2017). Prácticas inclusivas en la formación docente en México. Liberabit. Revista Peruana de Psicología 23(1), 39-56. https://doi.org/10.24265/liberabit.2017.v23n1.03

García, I., Romero, S., y Escalante, L. (2011). Diseño y validación de la guía de evaluación de las prácticas inclusivas en el aula GEPIA. Documento presentado en el XI Congreso nacional de investigación educativa.

http://www.comie.org.mx/congreso/memoriaelectronica/v11/docs/area_14/1390.pdf

García, I., Romero, S., Rubio, S., Jocabed, V., y Martínez A. (2015). Comparación de prácticas inclusivas de docentes de servicio de educación especial y regular en México. Revista Electrónica Actualidades Investigativas en Educación, 15(3), 1-17. http://dx.doi.org/10.15517/aie.v15i3.20671

Gómez, L., Castillo, R., Camacho, A., Sánchez Y., y De La Peña, C. (2017) Análisis de las actitudes y prácticas inclusivas en República Dominicana. Revista Nacional e Internacional de Educación Inclusiva, 10(1), 192-193. https://revistaeducacioninclusiva.es/index.php/REI/article/view/275 
Granada, M., Pómes, M., y Sanhueza, S. (2013). Actitud de los profesores hacia la inclusión educativa. Papeles de trabajo - Centro de Estudios Interdisciplinarios en Etnolingüística y Antropología Socio-Cultural,

http://www.scielo.org.ar/scielo.php?script=sci_arttext\&pid=S185245082013000100003\&lng=es\&tlng=es

Hernández, R., Fernández, C., y Baptista, P. (2014). Metodología de la investigación (6º ed.). México: Editorial Mc Graw-Hill.

Hernández , B., y García, I. (2017). Evaluación de actitudes, conocimientos y prácticas de educación inclusiva en docentes de primaria. Congreso Nacional de Investigación Educativa (COMIE).

http://www.comie.org.mx/congreso/memoriaelectronica/v14/doc/1382.pdf

Marchesi, A., Durán, D., Giné, C., y Hernández, L. (2009). Guía para la reflexión y valoración de prácticas inclusivas. Organización de Estados Iberoamericanos para la Educación, la Ciencia y la Cultura (OEI) https://www.oei.es/historico/inclusivamapfre/Guia.pdf.

Mateus, L., Vallejo, D., Obando, D., y Fonseca, L. (2017). Percepción de las prácticas y de la cultura inclusiva en una comunidad escolar. Avances en Psicología Latinoamericana, 35(1), 177-191. http://dx.doi.org/10.12804/revistas.urosario.edu.co/apl/a.4854

Ministerio de Educación. (2009). Decreto Supremo 170 de 2009: Fija normas para determinar los alumnos con necesidades educativas especiales que serán beneficiarios de las subvenciones para educación especial. Diario Oficial de la República de Chile. https:/especial.mineduc.cl/wp-content/uploads/sites/31/2018/06/DTO-170_21-ABR2010.pdf

Ministerio de Educación. (2015). Decreto N83 de 2015: Aprueba criterios y orientaciones de adecuación curricular para estudiantes con necesidades educativas especiales de educación parvularia y educación básica. Diario Oficial de la República de Chile. http://especial.mineduc.cl/wp-content/uploads/sites/31/2016/08/Decreto-83-2015.pdf

Ministerio de Educación. (2018). Decreto $N^{\circ} 67$ de 2018: Aprueba normas mínimas nacionales sobre evaluación, calificación y promoción y deroga los decretos exentos $N^{\circ} 511$ de 1997, $N^{\circ}$ 112 de 1999 y $n^{\circ} 83$ de 2001, todos del Ministerio de Educación. Diario Oficial de la República de Chile. https://www.bcn.cl/leychile/navegar?idNorma=1127255

Ministerio de Educación. (2017). Orientaciones sobre estrategias diversificadas de enseñanza para Educación Básica, en el marco del Decreto 83/2015. https:/especial.mineduc.cl/wpcontent/uploads/sites/31/2017/05/ORIENTACIONES_D83_Web_05-2017.pdf

Molpeceres, M., Chulvi, B., y Bernad, J. (2004). Concepciones sobre la enseñanza y prácticas docentes en un sistema educativo en transformación: un análisis en los PGS.

Organización Internacional del Trabajo. Centro Interamericano de investigación y documentación sobre formación docente.

80 | INTEREDU № 3 VOL. II (DiCIEMBRE 2020) PÁGS. 61-81. ISSN: 2735-6523 
http://www.scielo.org.co/scielo.php?script=sci_nlinks\&ref=000123\&pid=S0123$1294200900020000700009 \& \operatorname{lng}=\mathrm{en}$

Otzen, T., y Manterola, C. (2017). Técnicas de muestreo sobre una población a estudio. International Journal of Morphology, 35(1), 227-232. http://dx.doi.org/10.4067/S071795022017000100037

Rayo, A., y Pereyra, J. (2015). Actitudes ante prácticas inclusivas en Educación. Revista Científica De FAREM-Estelí, (13), 70-74. https://rcientificaesteli.unan.edu.ni/index.php/RCientifica/article/view/610

Rodríguez, G., Gil, J., y García, E. (1999). Metodología de la investigación cualitativa. Granada: Ediciones Aljibe.

Rubio, S. (2015). La participación colaborativa de las familias a través de las prácticas inclusivas (Tesis de maestría). Universidad de Valladolid. https://nanopdf.com/download/tfm-b96pdf_pdf

Ruiz, M., Borboa, M., y Rodríguez, J. (2013). El enfoque mixto de investigación en los estudios fiscales. TLATEMOANI Revista Académica de Investigación. (13), 1-25. http://www.eumed.net/rev/tlatemoani/13/estudios-fiscales.pdf

Sales, A., Moliner, O., Traver, J., García, R., Moliner, L., Oliver, R... y Ruiz, P. (2010). La construcción de la escuela intercultural inclusiva desde procesos de investigación-acción. servicio de Publicaciones de la Universitad Jaume I.

http://www.jmunozy.org/files/NEE/tutorias/inclusiva/jornadas_escuela_inclusiva/ materiales/ODET_MOLINER_Y_LIDON_MOLINER/Escuela_Interc_Inclusiva.pdf

UNESCO (2017). Guía para asegurar la inclusión y la equidad en la educación. Organización de las Naciones Unidas para la Educación, la Ciencia y la Cultura. http://down21chile.cl/cont/cont/2017/336_2_guia_para_asegurar_la_inclusion_y_la_equidad_en_1 a_educacion.pdf 